\title{
3-D granular modeling and in situ X-ray tomographic imaging: A comparative study of hot tearing formation and semi-solid deformation in $\mathrm{Al}-\mathrm{Cu}$ alloys
}

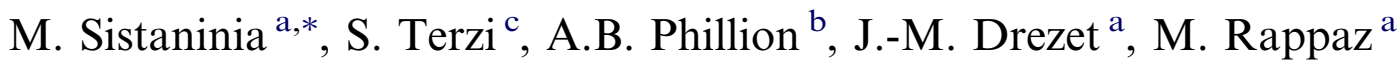 \\ ${ }^{a}$ Computational Materials Laboratory, Ecole Polytechnique Fédérale de Lausanne, Lausanne, Switzerland \\ ${ }^{\mathrm{b}}$ Okanagan School of Engineering, University of British Columbia, Kelowna, Canada \\ ${ }^{\mathrm{c}}$ European Space Agency, ESTEC, ILL4, 6 rue Jules Horowitz, 38042 Grenoble Cedex, France
}

Received 14 December 2012; received in revised form 18 March 2013; accepted 18 March 2013

Available online 17 April 2013

\begin{abstract}
The mechanical behavior of partially solidified $\mathrm{Al}-\mathrm{Cu}$ alloys is investigated to assess the influence of mushy zone deformation on hot tearing. For this purpose, the results of a semi-solid tensile test conducted in situ using X-ray microtomography are compared with the predictions of a coupled hydromechanical granular model in order to both validate the predictions of the model and explain the experimental observations. It is shown that hot tears initiate in the widest liquid channels connected to the free (oxidized) surfaces as long as there is contact between the intergranular liquid and the ambient air. The necking behavior is associated with the deformation-induced liquid pressure drop. Overall, the stresses predicted by the granular model under tensile and shear deformations agree well with the experimental data. Thus, the granular model achieves an important step in predicting hot tearing formation.
\end{abstract}

(C) 2013 Acta Materialia Inc. Published by Elsevier Ltd. All rights reserved.

Keywords: Microstructure; Hydromechanical coupling; Semi-solid; In situ tomography; Hot tearing

\section{Introduction}

During solidification, metallic alloys undergo deformation due to both solidification shrinkage and thermal contraction. If the deformation cannot be compensated by liquid flow, the liquid pressure drops even to negative values when a gas phase cannot nucleate. ${ }^{1}$ On the other hand, pressure present in the liquid induces a rearrangement of the solid grains if they are not too tightly packed. Thus, the liquid feeding and the ability of the solid skeleton to contract within the mushy zone dictate the maximal pressure drop within the liquid [2]. If the liquid pressure falls

\footnotetext{
* Corresponding author. Tel.: +41 216933943.

E-mail address: meisam.sistaninia@a3.epfl.ch (M. Sistaninia).

${ }^{1}$ Gas-free liquids can withstand considerable negative pressure because of the cohesive forces acting between the molecules [1]. However, in metallic alloys, which always have impurities and inclusions, the liquid pressure drop is lessened by pore formation.
}

below a cavitation pressure, a void may form and give rise to a hot tear [3]. Understanding the constitutive behavior of semi-solid alloys is thus essential for hot tearing prediction.

Many hot tearing theories and models have been developed during the past few decades to predict and better control the appearance of this very detrimental defect in casting and welding (see the review by Eskin et al. [4]). Early theories were based on strain accumulation in hot spot regions. Subsequent studies suggested that the critical parameter for hot cracking is not strain, but strain rate. Recently, Sistaninia et al. [2] demonstrated that the dependence of hot tearing on strain or strain rate is linked to the feeding condition of the mushy zone. When the mushy zone is able to be fed sufficiently, strain rate is the main critical factor for hot tearing. However, when the mushy zone is not fed sufficiently, hot tearing formation will also be affected by strain accumulation. The authors also 
showed that the primary stresses developed in metals during solidification are mainly due to liquid pressure drop; and not due to the contraction of the primary phase frequently suggested by other authors.

Most recently developed criteria, such as the RDG criterion [3], consider the pressure drop due to shrinkage and deformation as the main cause of hot tearing. Based on this theory, a hot tear does not form as long as the mush is fed sufficiently by melt flow during solidification. More recently, many efforts have been devoted to developing a mathematical model for hot tearing prediction. In this regard, the semi-solid behavior appears to be quite complex in comparison with solid deformation and fluid flow.

It has been experimentally observed that partially solidified alloys exhibit the characteristics of a granular material $[5,6]$. Over the past few years, a number of studies have been conducted that apply the concepts of granular mechanics to the behavior of solidifying alloys [7-13]. Compared to continuous average methods [3,14-17], granular approaches can account for the localization of strains and feeding in semi-solid materials, as well as for the stochastic aspects of hot tearing. Recently, a three-dimensional (3-D) coupled hydromechanical granular model [2] has been developed for equiaxed globular grains. Based on a Voronoi tessellation of randomly distributed nucleation sites, this model is able to predict the overall response of the semi-solid alloys to an externally applied tensile strain before and after fracture initiation, while accounting for the localization of strains at grain boundaries.

On the other hand, highly focused and intense X-ray beams available at synchrotron facilities and in the laboratory have started to play an important role in the investigation of semi-solid alloys (e.g. [5,18-20]). In terms of deformation behavior, the use of X-ray microtomography allows for detailed observation of the 3-D liquid/solid configurations, the propagation of hot tears and the interactions of hot tears with the microstructure. Besides digital image post-processing (DIP) of tomography images, volumetric digital image correlation can also be applied to obtain a sequence of incremental 3-D displacement fields of a deforming specimen.

In the present study, an extensive validation of the new hydromechanical granular model developed by Sistaninia et al. [2] is undertaken, specifically at the level of the grains, by comparison with previously conducted semi-solid tensile tests imaged in situ and in three dimensions by Terzi et al. [18] using X-ray microtomography at the European Synchrotron Radiation Facility (ESRF). This comparison allows for new perspectives of the study of the deformation behavior of semi-solids. First, the main features of the hydromechanical granular model are recalled for the sake of completeness. Second, the experimental methodology for acquiring in situ 3-D images of semi-solid deformation is presented. Finally, the predictions of the granular model are compared against the experimental results.

\section{Model development}

The hydromechanical granular model for semi-solid deformation consists of four separate 3-D modules: (i) a solidification module (SM) for generating the solid-liquid geometry at a given solid fraction; (ii) a fluid flow module (FFM) for pressure drop calculation; (iii) a semi-solid deformation module (SDM) for localization of straining; and (iv) a failure module (FM) for modeling fracture development. Since solid deformation, intergranular flow and crack initiation are deeply linked, the FFM, SDM and FM are fully coupled. This is achieved by carrying out each of the FFM, SDM and FM simulations incrementally, with iterations between each increment, until bulk fracture occurs. The reader is referred to Ref. [2] for an extensive description of the model; only a salient description is provided below.

\subsection{Solidification module}

In the SM, grain nuclei are first distributed randomly within a domain of uniform temperature. Nucleation is assumed to occur simultaneously, at the liquidus temperature. The grain structure is assumed to remain globular, thus the final grain boundaries correspond to the Voronoi tessellation of the random nucleation centers. Each polyhedral grain derived from the Voronoi tessellation is divided into a set of pyramids, as shown in Fig. 1b. These pyramids are further divided into tetrahedral elements by subdividing each polygonal face into triangles (i.e. Fig. 1c). Solidification is approximated within each tetrahedron using a microsegregation model with infinite diffusion in the liquid and some back-diffusion in the solid. Once two solid-liquid interfaces are within interaction distance, a coalescence model is activated, thus leading to the formation of grain clusters (a group of grains which are mechanically linked).

\subsection{Fluid flow module}

The FFM solves the simplified Poiseuille flow equation due to the geometry of the liquid channels that remain in

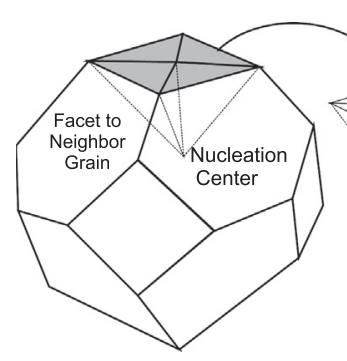

(a)

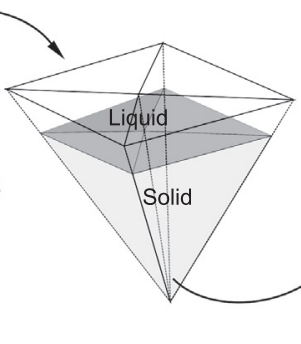

(b)

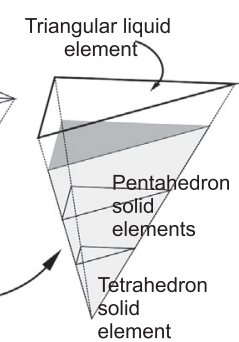

(c)
Fig. 1. Schematic of the granular model mesh structure: (a) a polyhedral grain as computed with the Voronoi tessellation; (b) a pentahedral volume element showing both the solid portion (grey) and the liquid portion (clear); (c) a single tetrahedron decomposed into a set of solid elements. 
between the solidifying polyhedral grains [11]. When combined with the conservation of mass, the Poiseuille flow equation in each liquid channel gives

$\frac{2 h^{3}}{3 \mu_{\ell}} \nabla^{2} p_{\ell}=2 \beta v^{*}+\Delta v_{s n}+\frac{2 h}{K_{\ell}} \frac{\partial p_{\ell}}{\partial t}$

where $p_{\ell}$ is the liquid pressure, $h$ is the half-width of the liquid channel, $\mu_{\ell}$ is the dynamic viscosity of the liquid, $\beta=\left(\rho_{s} / \rho_{\ell}-1\right)$ is the shrinkage factor, $\rho_{\ell}$ and $\rho_{s}$ are the densities of the liquid and solid, respectively, $K_{\ell}$ is the bulk modulus of the liquid, $\Delta v_{s n}$ is the difference between the normal velocities of the two adjacent grains calculated by the SDM and $v^{*}$ is the solidification velocity of the solid-liquid interface calculated by the SM. Eq. (1) is solved using the finite element method. Due to the flow being parallel to the facets, the 3-D prismatic geometry is discretized into three-node 2-D triangular elements.

\subsection{Semi-solid deformation module}

The SDM utilizes a combined finite element/discrete element methodology to predict the deformation behavior of semi-solid metallic alloys during solidification. Each solid tetrahedral element from the SM is split into three solid elements - a tetrahedron and two pentahedrons - as shown in Fig. 1c. The numerical simulation of the SDM is then performed using the commercial finite element code Abaqus 6.9TM. The mechanical behavior of the solid elements is assumed to be elasto-viscoplastic, with a flow stress based on the viscoplastic Ludwik's equation, while the resistance of the liquid films to separation prior to coalescence is assumed to be due only to the liquid pressure, $p_{\ell}$, from the FFM that is exerted on the solid-liquid interfaces. A frictionless hard-contact pressure-overclosure relationship is used to limit inter-grain penetration.

\subsection{Failure module}

Assuming that contact exists between the liquid and the atmosphere, which is the case of the semi-solid rheological tests $[16,18,21,22]$, the criterion for crack initiation and propagation can be estimated from the overpressure required to overcome capillary forces at the liquid-atmosphere interface. The hot crack starts to initiate in a liquid channel connected to the atmosphere once $p_{\ell}$ reaches a critical pressure $p_{\ell}^{c}$, given by [2]:

$p_{\ell}^{c}=p_{a}-\frac{\lambda \cos \Theta}{h}$

where $p_{a}$ is the atmospheric pressure, $\Theta$ is the dihedral angle and $\lambda$ is the surface tension at the void-liquid interface. Considering that a thin oxide skin forms between the liquid and atmosphere, the value of $\lambda \cos \Theta$ is fixed to $5 \mathrm{~J} \mathrm{~m}^{-2}$ in the present simulations. ${ }^{2}$ When the rate of deformation is

\footnotetext{
${ }^{2}$ For comparison, the non-oxidized liquid aluminum surface energy is about $1 \mathrm{~J} \mathrm{~m}^{-2}$.
}

very low, which is the case of Terzi's test [18], this value can also be used for modeling crack propagation since the oxide layer has enough time to form continuously during crack propagation. Consequently, the value of $5 \mathrm{~J} \mathrm{~m}^{-2}$ was used in the present study for the liquid/oxide-air interface during the entire time (several minutes) of Terzi's test. Otherwise, as explained in [2], when deformation occurs more rapidly, a smaller value based on a much less oxidized liquid aluminum surface can be used for crack propagation. This is why a value of $2 \mathrm{~J} \mathrm{~m}^{-2}$ was used for modeling propagation crack in the other simulations presented in Section 4.2.1.

\section{Experimental methodology}

The experimental work was carried out previously at ESRF Grenoble on the ID19 beamline by Terzi et al. [18]. The Al-8 wt. $\% \mathrm{Cu}$ alloy was cylindrical in shape, with a $2 \mathrm{~mm}$ diameter and a $8 \mathrm{~mm}$ length. Near the mid-height, the cross-section of the specimen was locally reduced to $1.5 \mathrm{~mm}$ in diameter to localize the tensile deformation to the region where the X-ray radiographic images were recorded. At the beginning of the test, the specimen was heated at a rate of $0.5 \mathrm{~K} \mathrm{~s}^{-1}$ by an induction coil up to $828 \mathrm{~K}$ and then held at this temperature. After a $3 \mathrm{~min}$ dwell time, the tensile test was initiated at a deformation rate of $0.1 \mu \mathrm{m} \mathrm{s}^{-1}$. A complete tomography scan was recorded every $27 \mathrm{~s}$, with a pixel size of of $2.8 \mu \mathrm{m}$.

At a temperature of $828 \mathrm{~K}$, the volume fraction of solid, $g_{s}$, is equal to 0.91 and 0.83 based on the lever-rule and Scheil equations, respectively. In comparison, the volume fraction of solid estimated from the 3-D image at the beginning of deformation was $g_{s}=0.93$, a value that is close to the equilibrium value given by the lever rule, indicating that the initial copper concentration was well homogenized in both the liquid and solid phases during isothermal holding.

\section{Results and discussion}

A description of the knowledge gained by the granular model simulations is first provided, then the distribution of liquid and pores in the simulations are compared against the experimental results from Terzi et al. [18]. Next, the stress-strain curves obtained from the simulation are compared against the results from other semi-solid tensile and shear experiments found in the literature. The material properties and physical parameters are those for $\mathrm{Al}-\mathrm{Cu}$ alloys, and can be found in Ref. [2].

\subsection{Simulation results}

The output of the hydromechanical granular model is shown in Figs. 2 and 3. In this simulation, only the central part of the tensile test specimen used by Terzi $(2 \mathrm{~mm}$ in length including the notched zone) is modeled. The upper side of the domain is axially fixed and the bottom side is 


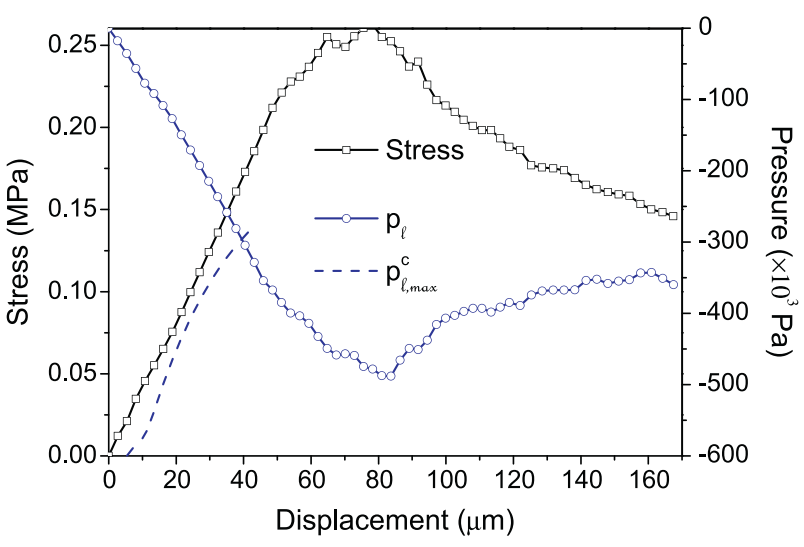

Fig. 2. Calculated tensile stress-strain behavior of the partially solidified tensile test sample [18] at $g_{s}=0.91$, as predicted by the granular model. The stress is given on the left axis while the liquid pressure is given on the right axis, both in relation to the axial displacement.

connected to a reference node, which is displaced at a fixed velocity of $v_{v}=0.1 \mu \mathrm{m} \mathrm{s}^{-1}$. The use of a reference node enables the bulk semi-solid mechanical behavior to be obtained directly from the calculated force-displacement curve at this location. The domain is free to deform radially. The solid fraction was set to $g_{s}=0.91$, matching the experimental value, and $p_{a}$ is assumed to be equal to zero. Furthermore, all of the surfaces of the domain are closed for the FFM calculation, simulating the unfeedable mushy zone situation seen in all of the semi-solid tensile tests $[16,18,21-23]$ that will be discussed.
The final critical parameter of the simulation is the average size of the grains, $d_{v}$. From many studies (e.g. [24]), it appears that hot tearing susceptibility of alloys increases with $d_{v}$. The present granular model also predicts that the mechanical resistance of semi-solid alloys to hot tearing decreases with increasing $d_{v}$ since the liquid channel widths for a given $g_{s}$ increase with $d_{v}$. The average liquid channel width, $2 h_{v}$, is linked to $d_{v}$ as

$2 h_{v} \sim d_{v}\left(1-g_{s}^{1 / 3}\right)$

Conversely, the liquid pressure required for void formation in a channel also increases with the liquid channel width, as shown by Eq. (2). In order for the simulation to correctly predict the crack formation in the test of Terzi, $d_{v}$ must be set so that the distribution of liquid channel widths is in agreement with the experimental data. Good agreement is found for $d_{v}=200 \mu \mathrm{m}$. It is difficult to verify this value as the average grain size of the specimen viewed in the tomography images is difficult to estimate for two reasons: the low resolution of the images (thin liquid channels might not be viewed) and the more complex globulardendritic morphology of the grains. Post-mortem electron backscatter diffraction measurements should have been done after the tomography experiments to obtain an unambiguous grain density, but such information was not available from this test. However, it seems that the grain size adopted in the simulation gives a repartition of the intergranular liquid which is similar to what is seen in the actual microstructure and corresponds to what is typical of

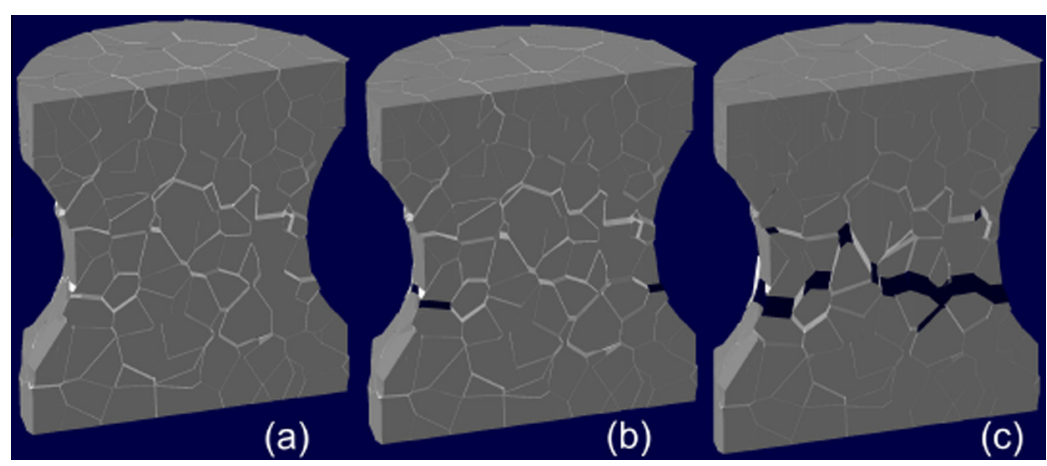

Fig. 3. Evolution in the semi-solid microstructure during the tensile deformation as predicted by the granular model at (a) $t=405 \mathrm{~s}\left(u_{v}=40.5 \mu \mathrm{m}\right)(\mathrm{b})$ $t=729 \mathrm{~s}\left(u_{v}=72.9 \mu \mathrm{m}\right)$ and $(\mathrm{c}) t=1215 \mathrm{~s}\left(u_{v}=121.5 \mu \mathrm{m}\right)$. The white areas outline the intergranular liquid, the dark areas the growing voids and the gray areas the solid phase.

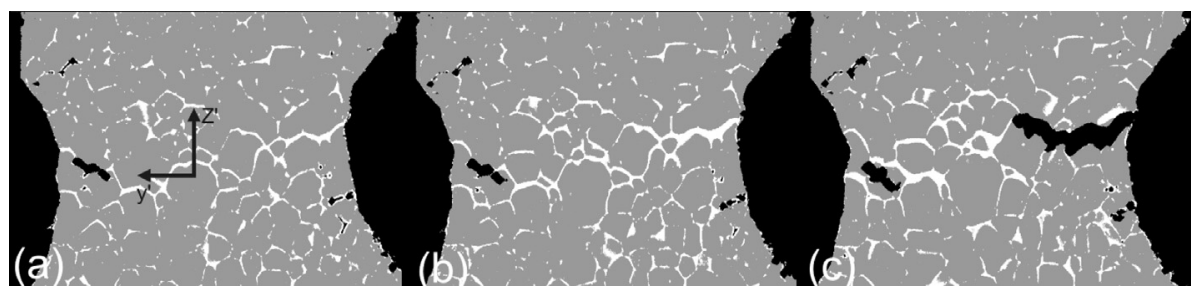

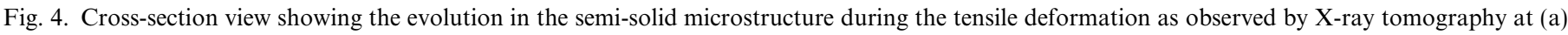

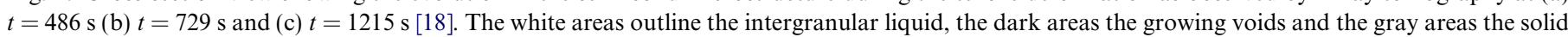
phase. 
inoculated $\mathrm{Al}$ alloys.

Fig. 2 shows the simulated tensile behavior of the semisolid material. The overall stress (the sum of the interaction forces imposed on the bottom side divided by the maximum cross-sectional area) is given on the left axis while the average liquid pressure drop $\bar{p}_{\ell}$ that occurs during the tensile deformation is given on the right axis, both in relation to the axial displacement, $u_{v}$. Although the pressure gradient through the liquid channel network is the driving force for the fluid flow in this network, it is negligible for the SDM and FM calculations due to the size of the domain, i.e. $\bar{p}_{\ell} \simeq p_{\ell}$. The failure pressure $p_{\ell, \text { max }}^{c}$ (output of FM) associated with the widest channel is also given (dashed curve).

The two curves $p_{\ell}$ and $p_{\ell, \max }^{c}$ cross at a displacement $u_{v}=40 \mu \mathrm{m}$. At this point, the simulation predicts that the first crack initiates in the widest channel connected to ambient air at the outer surface of the specimen. Although the stress within the domain continues to increase above this point until $\sigma_{v, \max }$, the rate $d \sigma_{v} / d u_{v}$ no longer increases. With continued deformation, other voids will initiate in the other channels connected to air. The voids then propagate and percolate together, leading to the overall failure of the specimen.

\subsection{Comparison with in situ observations}

Fig. 3 shows a sequence of three images of a longitudinal cross-section inside the simulation domain at three different times of deformation. The white areas outline the intergranular liquid, the gray areas show the solid phase and the dark areas are the growing voids. During tensile testing, liquid flows from the less deformed zone towards the notched zone in order to accommodate the deformation (i.e. there is drainage of liquid towards the most highly deformed region). Once there is no more liquid available for feeding, the voids nucleate and propagate. This occurs in the notched zone, since the widest channels are present in this part of the specimen owing to the accumulation of liquid, and this zone experiences the largest stress.

Fig. 4 shows a sequence of three X-ray images of the notched zone of the specimen at the same times of deformation as in Fig. 3. In this sequence, the accumulation of liquid and void propagation in the notched zone predicted by the granular model is clearly observed. In Fig. 5, the interfacial surfaces between the gas (void) and the solidliquid phases (specimen) in a top view of the sample are shown at different times of deformation. Although there are some initial voids (pores) inside the specimen at the beginning of deformation, all of the cracks initiate on the outer surface of the specimen. Comparing Fig. $5(t=0)$ and $(t=486)$, the initial voids that are not connected to the ambient air do not propagate; on the contrary, they disappear during the initial stages of deformation.

Fig. 6 shows a comparison of the distribution of the liquid channel widths as calculated by the granular solidification model (dashed curves) and measured on the experimental data using DIP (continuous curves) at $t=0$ and $t=729 \mathrm{~s}$. In order to measure the liquid channel widths, the solid-liquid interfaces of the 3-D geometry obtained by X-ray tomography were discretized into triangular elements. The width of each liquid film was then computed
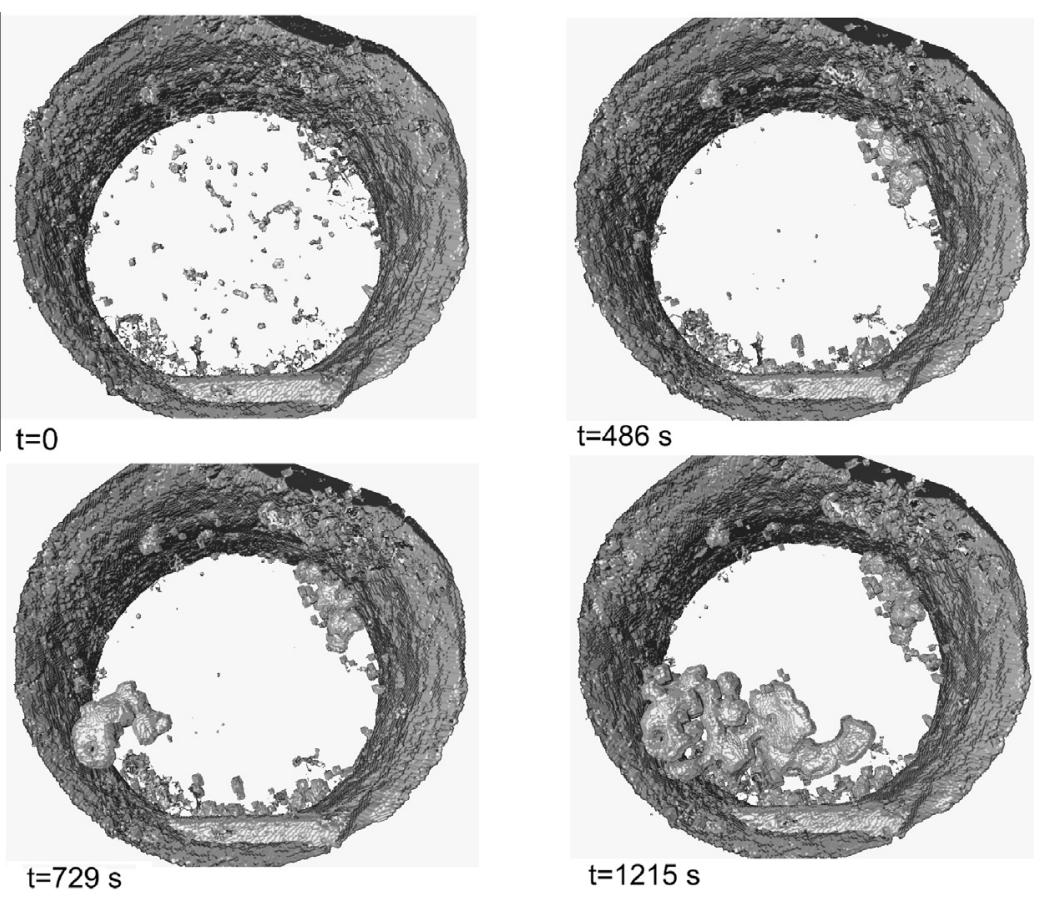

Fig. 5. Sequence of top view images of the sample, showing the interfacial surface between the atmosphere/void and the sample at four different times of deformation. 


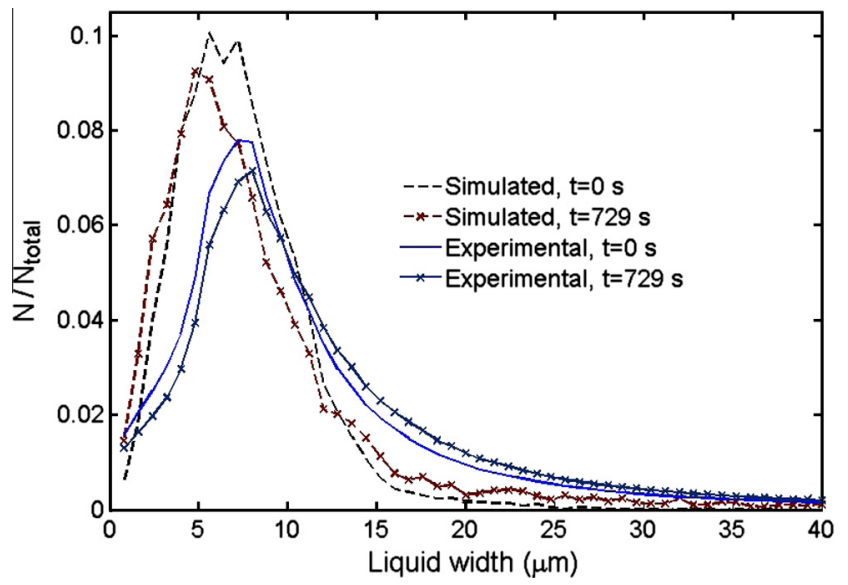

Fig. 6. Distribution of the liquid channel widths in the notched area of the specimen at $t=0 \mathrm{~s}$ and at $t=729 \mathrm{~s}$ as predicted by the granular model (dashed curves) and measured on the experimental data using the DIP technique (continuous curves).

based on the shortest distance of each vertex in the direction of its normal to all other triangular elements. The general trend predicted by the granular model is in a good agreement with the experimental data. Issues include:

- The width of the majority of the liquid channels is $\sim 8 \mu \mathrm{m}$.

- Although this value remains constant during deformation, the number of channels having this width decreases.

- The number of channels with width larger than $8 \mu \mathrm{m}$ increases during deformation.

- The width of channels which are perpendicular to the tensile axis tends to increase, while the width of those parallel to it tends to decrease as the grains come into closer contact during deformation.

Fig. 7 shows the variations of the area of the horizontal cross-section of the specimen along the tensile axis at various times of deformation as (a) predicted by the granular model and (b) measured on the experimental data. The vertical and horizontal axes of these curves are normalized by the current length of the notched zone (i.e. $\left.L=L_{0}(1+\varepsilon)\right)^{3}$ and the maximum cross-sectional area, respectively. The reference position for the normal axis is placed at the minimum cross-sectional area of the specimen, as shown in Fig. 4a, and only the upper half of the notched zone is considered $\left(0<\left(z^{\prime} / L\right)<0.5\right)$. The position of the minimum cross-section has been carefully determined based on the number of gray voxels (i.e. solid phase) and white voxels (i.e. liquid phase) in each horizontal-slice image.

As can be seen, the necking process predicted by the granular model is in good agreement with the experimental

\footnotetext{
${ }^{3}$ The current length of the notched zone of the specimen is measured on the X-ray tomography images.
}

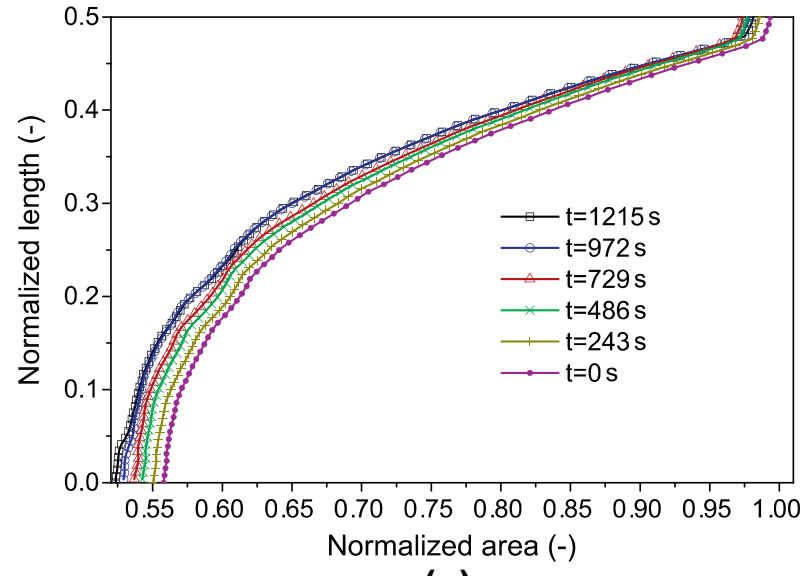

(a)

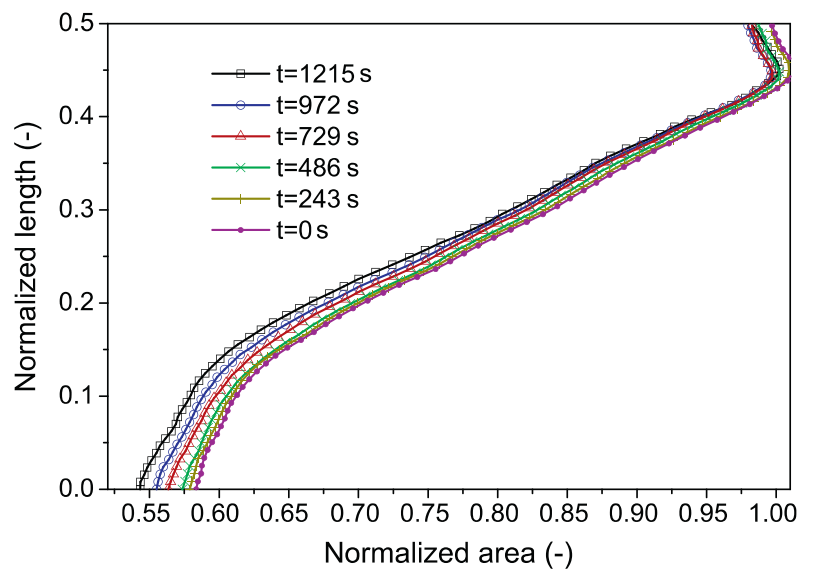

(b)

Fig. 7. The variation in horizontal cross-sectional area along the tensile axis during deformation as (a) predicted by the granular model and (b) measured on the experimental data using DIP technique. Only the results from the notched zone of the specimen are provided. The vertical tensile axis coordinate is normalized by the current length of the notched zone. The area is normalized by the maximum surface of the notched area.

data shown in Fig. 7b. Both the simulations and the experimental results indicate that the specimen continuously necks during tensile deformation and that the maximum necking occurs at the minimum cross-sectional area of the specimen, i.e. at $z^{\prime}=0$. As explained before, since the deformation cannot be compensated by liquid feeding, the liquid pressure drops due to the extension of the solid along the vertical $z$-axis. The negative intergranular liquid pressure forces the domain to contract in order to minimize its volume change. However, the overall compressibility of the semi-solid alloys decreases and approaches the incompressibility of a fully solid structure $\left(g_{s}=1\right)$ as the liquid in the channels parallel to the tensile axis flows out and the grains come into contact with each other. This decrease in compressibility leads to the increase in the rate $-d p / d u_{v}$ and consequently the increase in the rate $d \sigma_{v} / d u_{v}$ [2]. After $t=800 \mathrm{~s}$ (displacement $80 \mu \mathrm{m}$ ), which corresponds to the minimum liquid pressure (see Fig. 2), necking of the 


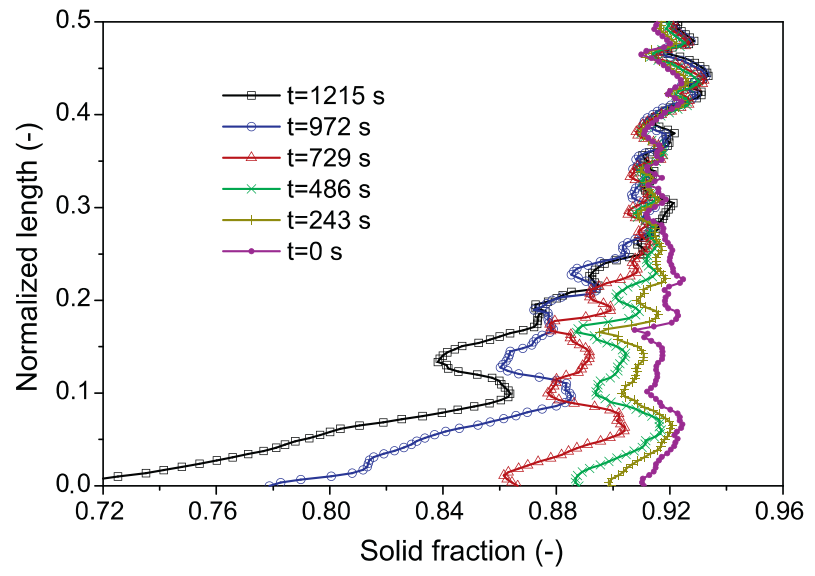

(a)

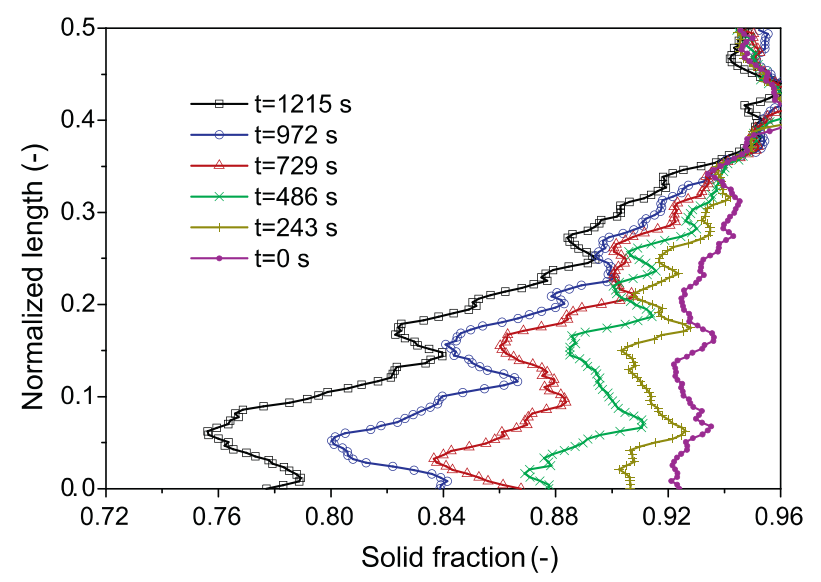

(b)

Fig. 8. Variation in the solid fraction along the tensile axis during deformation as (a) predicted by the granular model and (b) measured using DIP technique. Only the results from the notched zone of the specimen are provided. The vertical tensile axis coordinate is normalized by the current length of the notched zone.

specimen is almost frozen. Because of that, the two curves at $t=972 \mathrm{~s}$ and $t=1215 \mathrm{~s}$ are almost superimposed.

Fig. 8 shows the variations of the solid fraction along the tensile axis at various times of deformation as (a) predicted by the granular model and (b) measured on the experimental data. The tensile axis coordinate is again normalized by the length of the notched zone. As can be seen in Fig. 8a, $g_{s}$ is rather uniform along the tensile axis at the beginning of deformation. However, the accumulation of liquid in the middle of the notched zone with increasing deformation leads to a decrease in $g_{s}$ in this region while a small increase in $g_{s}$ is observed near the end of the notched zone. A similar trend is observed in the experimental result (Fig. 8b) except that an axial solid fraction gradient is present at the beginning of deformation, indicating that a thermal gradient is present along the tensile axis.

Fig. 9 shows the variation in void fraction, $g_{p}$, along the tensile axis at various times of deformation as (a) predicted by the granular model and (b) measured on the experimental data. Since, in the simulation, the very first voids form

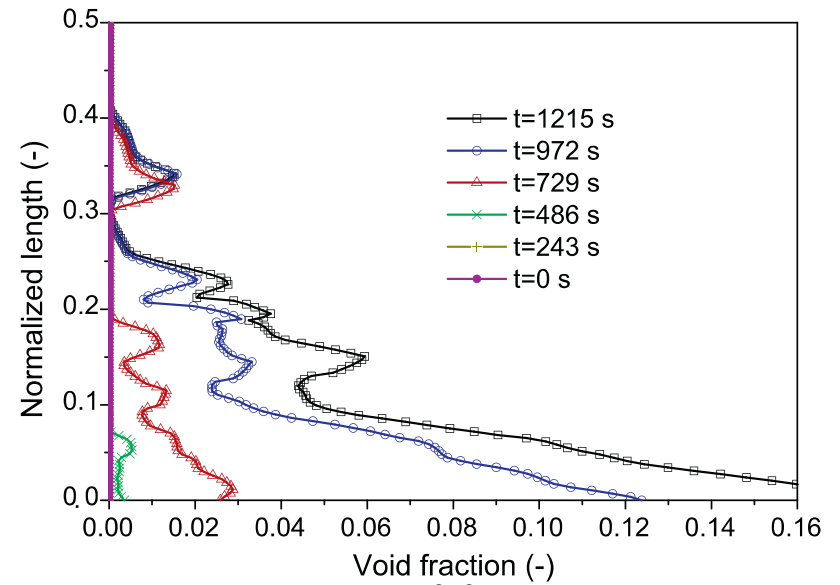

(a)

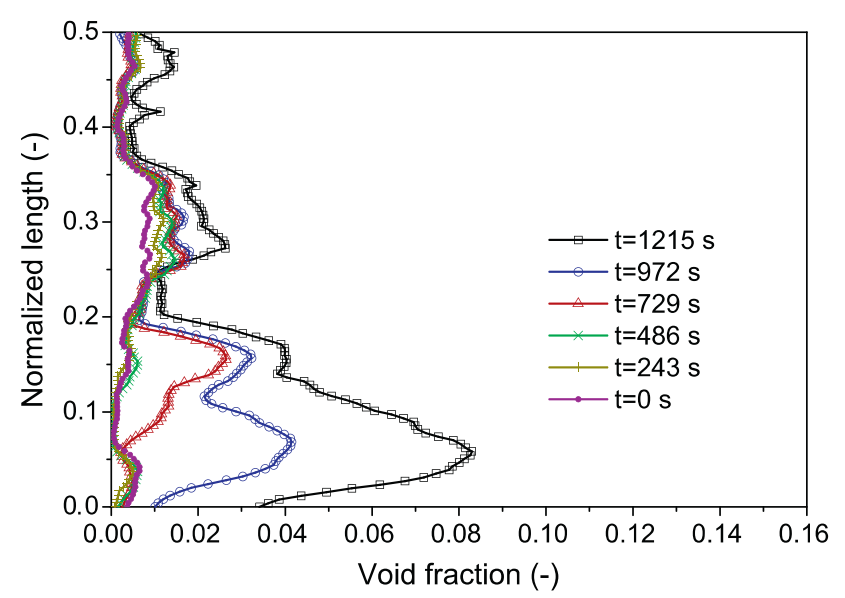

(b)

Fig. 9. Variation in the void fraction along the tensile axis during deformation as (a) predicted by the granular model and (b) measured using DIP technique. Only the results from the notched zone of the specimen are provided. The vertical tensile axis coordinate is normalized by the current length of the notched zone.

at $t=400 \mathrm{~s}, g_{p}$ is zero for the two curves $t=0 \mathrm{~s}$ and $t=243 \mathrm{~s}$. In contrast, some voids are present in the experimental data at $t=0 \mathrm{~s}$. Experimentally, the voids that are connected to the ambient air propagate slightly during deformation, while those which are not connected to air disappear. Although the granular model predicts the time of crack initiation accurately, it overestimates the rate of crack propagation as the maximum $g_{p}$ calculated at $t=1215 \mathrm{~s}\left(g_{p, \max }=0.17\right)$ is about twice the measured value. This discrepancy could be related to the shape of the grains, which in the present simulation is assumed to be a polyhedron, whereas in the specimen it could be somewhat dendritic. However, the discrepancy could also be explained if one further analyzes the 3-D tomographic images in order to determine the change in solid and liquid fractions during the tensile deformation.

Fig. 10 shows the normalized liquid volume change that occurs in the upper half of the notched zone as a function of displacement, as well as the product of the normalized 


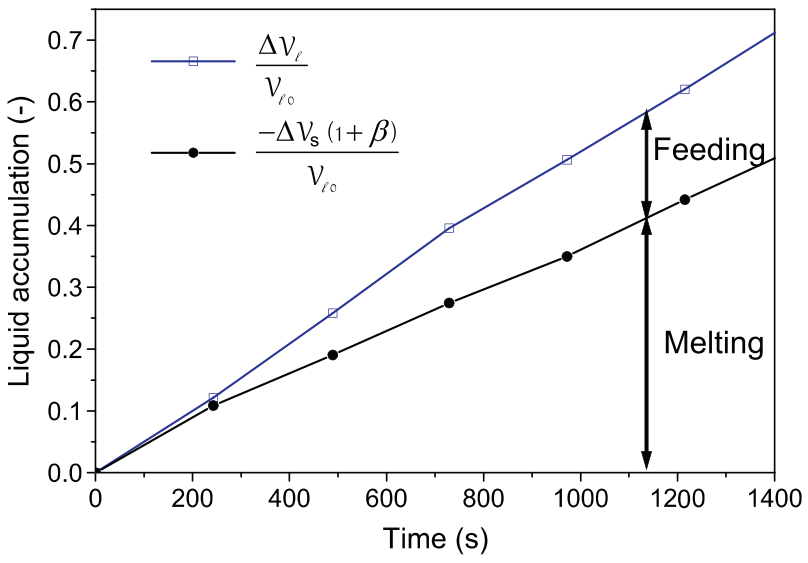

Fig. 10. Accumulation of liquid in the notched zone $\left(\frac{\Delta v_{\ell}}{v_{\ell 0}}\right)$ during tensile deformation and due to both melting $\left(\frac{-\Delta v_{s}(1+\beta)}{v_{\ell 0}}\right)$ and feeding.

solid volume change and the factor $(1+\beta)$, with the opposite sign. Both curves have been normalized against the initial liquid volume. In order to be meaningful, it is necessary to make such mass balance - or volume balancing, if one accounts for the density variations - over a Lagrangian domain. Since X-ray tomography is typically an Eulerian visualization of a specimen (fixed window), the following procedure was adopted to obtain Fig. 10.

First, it was confirmed that the upper side of the notched zone did not move axially during deformation. For this purpose, the position of a flaw near the upper side of the notched zone was checked in the tomography images at different times. Second, the position of the minimum crosssection was carefully determined, as explained above. This mid-section of the specimen is the symmetric plane of the specimen; hence the flux of matter across it should be zero. Since the lateral surfaces of the specimen are also isolated, any change in mass within the volume bounded by the upper and mid-section surfaces has to come from the upper surface. Third, the numbers of gray and white voxels within the considered Lagrangian volume were counted to calculate the volume of the solid and liquid phases in the upper half of the notched zone. Since the solid and liquid densities are constant, volume changes correspond to mass changes once the shrinkage factor $\beta$ is considered.

As deformation proceeds, the volume of liquid in the upper part of the notched zone is seen to increase. This volume increase partially occurs due to fluid flow from the upper surface of the considered volume, since part of the specimen is not imaged by X-ray tomography. If the accumulation of liquid in the deformed region is only due to the feeding, the volume of solid should remain constant. However, as can be seen, the volume of solid decreases, which seems to indicate that the solid phase partially melts during tensile deformation. In this case, the product of the solid volume change and $(1+\beta)$ gives the additional amount of liquid created due to partial melting. This partial melting can be attributed to:
1. An increase in the portion of the upper half of the notched zone that falls within the hot zone of the setup, as the temperature is certainly not uniform along the tensile axis due to the tensile machine microgrips.

2. Localized remelting induced by liquid feeding, since the liquid coming within the analyzed volume is richer in $\mathrm{Cu}$ as it comes from a colder zone (i.e. solutal remelting).

Partial melting during tensile deformation of semi-solid alloys was also previously observed during in situ X-ray radiography experiments on $\mathrm{Al}-\mathrm{Cu}$ alloys [25]. The liquid volume increase due to partial melting (and feeding) provides an explanation for the lower crack propagation rate seen in the experimental data as compared with the simulation result. The predictions could be improved by modeling the whole specimen instead of only two millimeters of it, as the rest of the specimen could participate in the feeding of the notched zone.

The liquid accumulation in the deformed region may also explain the disappearance of the initial pores in this region during the tensile deformation, since the liquid phase can hold a larger amount of dissolved gases than the solid.

Alternatively, the solid volume change may also be linked to the appearance, during deformation, of liquid channels that are too thin to be detected by X-ray tomography at the beginning of deformation. If one assumes that the liquid channels thinner than one pixel size are not detected, the granular solidification model estimates that $14 \%$ of the liquid is not detected at $t=0$.

\subsubsection{Comparison with rheological tests}

The load exerted on the tensile sample during the in situ X-ray tensile deformation was too low $\left(F_{\max }=0.78 \mathrm{~N}\right.$, predicted by the present model) to be measured due to the very small size of the specimen. Nevertheless, in Figs. 11-13, a quantitative validation of the failure stresses predicted by the hydromechanical granular model is presented, by comparing the calculated maximum stress for different $g_{s}$ to

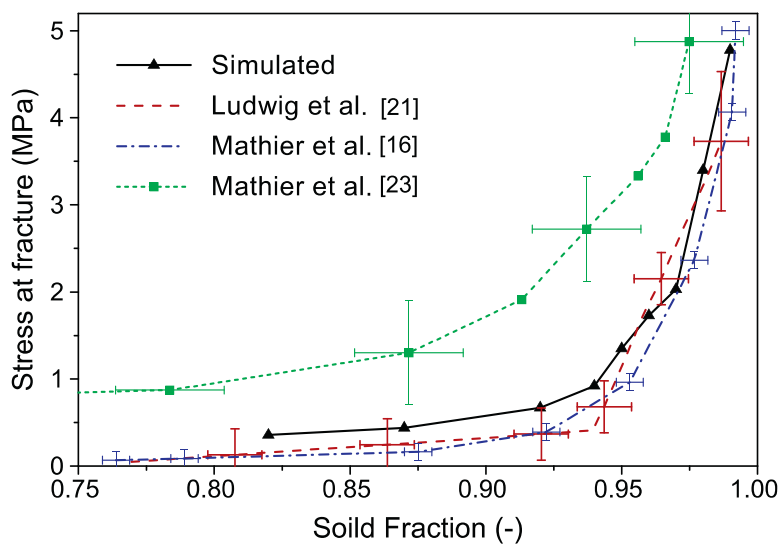

Fig. 11. Comparison between the tensile strength measured in a semi-solid Al-2 wt.\% Cu alloy (dashed line curve) by Ludwig et al. [21] and Mathier et al. [16,23], and the results of the granular model (continuous curve). 


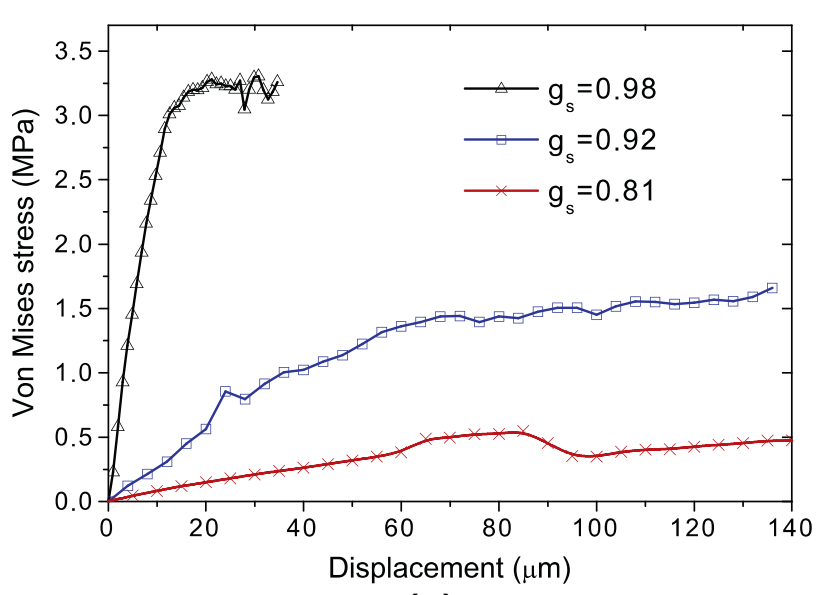

(a)

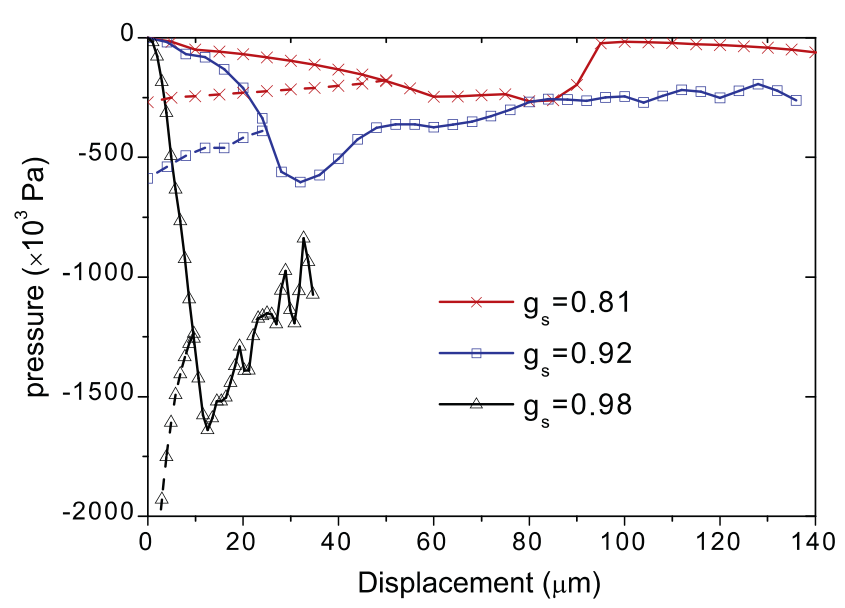

(b)

Fig. 12. Shear behavior of a partially solidified $\mathrm{Al}-2 \mathrm{wt} . \% \mathrm{Cu}$ alloy for various fractions of solid: (a) stress vs. displacement curves, (b) pressure vs. displacement.

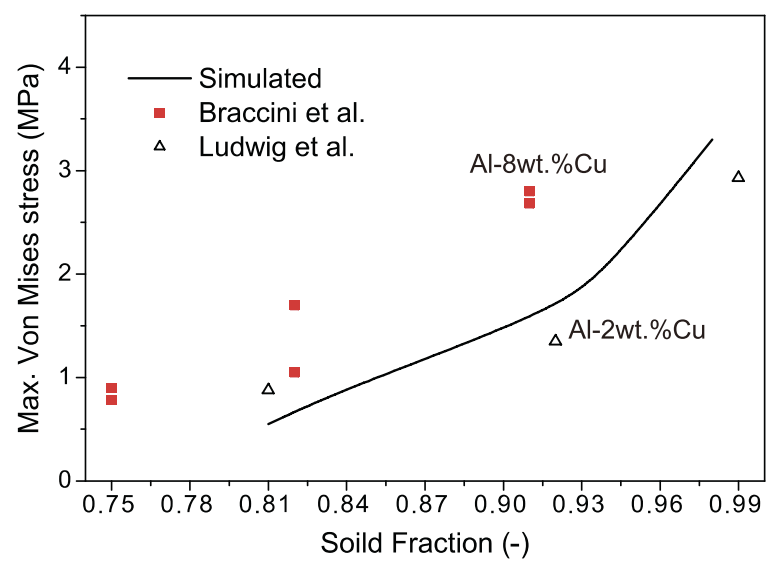

Fig. 13. Comparison between the shear strength measured in semi-solid $\mathrm{Al}-\mathrm{Cu}$ alloys by Ludwig et al. [21] and Braccini et al. [22], and the results of the granular model.

experimental results available in the literature. For this validation, the simulations were performed using a 1000-grain cubic representative volume element $(\mathrm{RVE})(10 \times 10 \times 10)$ with a grain size of $100 \mu \mathrm{m}$ over a range of solid fraction between 0.8 and 0.99 . Note that the size of the grains was not measured in the experimental tests. The average size of the grains was fixed to $100 \mu \mathrm{m}$ since grains of this size are expected to be globular [26]. The effect of $\lambda \cos \Theta$ on $\sigma_{\max }$ has been shown in Ref. [2]. One can estimate the effect of grain size on $\sigma_{\max }$ considering the findings along with Eqs. (3) and (2).

Fig. 11 provides a comparison between the simulated maximum stress (stress at fracture) and the experimental semi-solid failure stress data for an $\mathrm{Al}-2 \mathrm{wt} . \% \mathrm{Cu}$ alloy measured by Ludwig et al. [21] and Mathier et al. [16,23] at a strain rate of $10^{-3} \mathrm{~s}^{-1}$. As can be seen in the figure, the tensile strength measured with the experimental device developed by Mathier et al. [23] is significantly higher than the values measured with two other apparatus [16,21]. There is first an offset of about $0.7 \mathrm{MPa}$ measured at low $g_{s}$ in the device of Mathier et al. [23], which is probably due to the compliance of the set-up itself. Subtracting this "residual" strength from all the data points of Mathier et al. [23], all the experimental data are in fairly good agreement with each other. More importantly, it can also be seen in this figure that the model accurately predicts the strength of semi-solid alloys with a large solid fraction. However, for $g_{s}<0.94$, it seems that this model somewhat overestimates the alloy strength compared to the experiments.

Fig. 12a shows the simulated rheological behavior of the semi-solid RVE under shear deformation for three values of $g_{s}(0.92,0.96$ and 0.98$)$. In this case, the semi-solid deformability, i.e. the amount of strain for a given stress, is higher compared to that of a tensile test (Fig. 11). This phenomenon is due to the fact that, while the intergranular liquid films do not allow two grains to be separated in tension without liquid feeding, they do allow the grains to slide across each other since no volume change is involved. Experimental results for aluminum alloys also demonstrate different semi-solid tension and shear mechanical behaviors. Specifically, it has been shown that the volume of granular semi-solid materials increases during shear deformation due to Reynolds dilatancy [5], which indicates that the liquid pressure of an unfeedable RVE must reduce in shear deformation. This pressure drop during shear deformation in a way similar to tensile deformation leads to hot tearing formation.

In Fig. 12b, the intergranular liquid pressure drop during the shear deformation is reported (continuous curves), along with the $p_{\ell, \max }^{c}$ value (dashed curves). As can be seen, the liquid pressure is correctly predicted to be negative during shear deformation. Furthermore, it decreases as deformation proceeds until a minimum value is reached. Although the liquid pressure then increases, the overall stress remains nearly constant. In comparison, the stress decreases after reaching a maximum value in tensile deformation. Previous experimental works have also shown that the stress-strain curves for semi-solid alloys in shear deformation end with a plateau $[21,22]$. 
Finally, in Fig. 13, a comparison of the semi-solid shear behavior (von Mises stress) is made between the simulation predictions and the experimental results for an Al-2 wt.\% $\mathrm{Cu}$ alloy [21] and an $\mathrm{Al}-8 \mathrm{wt} . \% \mathrm{Cu}$ alloy [22]. The simulation have been done with a 2 wt.\% $\mathrm{Cu}$ composition, but comparing with the experimental results at the corresponding fraction of solid. Although the simulation results do not match either experimental result, the trends are a good match (and the scale is quite small). Furthermore, there is considerable variation in the experimental data. The deviation between the experimental results could be due to a number of factors: (i) the no-slip condition experimentally assumed to exist at the cylinder surface by Ludwig et al. [21] might not be respected, whereas grooves were machine on the surface of the inner and outer cylinders used by Braccini et al. [22] in order to avoid slippage at the interface; (ii) in the experimental tests of Braccini et al. [22], only a few grains ( $\sim 6$ grains) were present along the radius due to the small gap between the two cylinders. It has been demonstrated that the deformability of a small RVE containing a few grains decreases with the number of grains present in the RVE [27]; (iii) the difference in the $\mathrm{Cu}$ content might influence both the solid fraction and, to a lesser extent, the rheological parameters; and (iv) the size of the grains in the experimental tests of Braccini et al. [22] is significantly larger than in the work of Ludwig et al. [21]. This implies that the shape of the grains in the tests of Braccini et al. [22] is dendritic; consequently, the effective solid fraction is higher than what is shown in Fig. 13 since it accounts for only the intergranular liquid.

\section{Conclusion}

The results of a hydromechanical granular model have been compared on the one hand with in situ X-ray tomographic observations made during the tensile deformation of a mushy $\mathrm{Al}-\mathrm{Cu}$ alloy specimen, and on the other hand with the overall rheological behavior of semi-solids measured by various mechanical tests. It has been shown that, during tensile testing, fluid flows toward the deformed region to compensate for deformation. Furthermore, the specimen continuously necks, allowing the liquid channels perpendicular to the tensile axis to open by feeding them with the liquid coming from neighboring zones and from channels which are parallel to the tensile axis and tend to close. Once the liquid is no longer able to feed the deformed zone, cracks form in the structure. The granular model also demonstrates that the grain size has a large effect on the 'overpressure' required to overcome the capillary forces at the liquid-void interface. Because of this dependence, the hot tearing susceptibility increases with increasing grain size.

\section{Acknowledgements}

The authors thank the Swiss Competence Centre for Materials Science and Technology (CCMX) and partner companies within the thematic area "Multi-scale, multiphenomena modelling of metallic systems" for funding this research.

\section{Appendix A. Supplementary material}

Supplementary data associated with this article can be found, in the online version, at http://dx.doi.org/10.1016/ j.actamat.2013.03.021.

\section{References}

[1] Hoyt JJ, Potter AA. A molecular dynamics simulation study of the cavitation pressure in liquid al. Metall Mater Trans A 2012;43:3972-7.

[2] Sistaninia M, Phillion AB, Drezet JM, Rappaz M. A 3d coupled hydro-mechanical granular model for constitutive behavior of metallic alloys during solidification. Acta Mater 2012;60:6793-803.

[3] Rappaz M, Drezet JM, Gremaud M. A new hot-tearing criterion. Metall Mater Trans A 1999;30:449-55.

[4] Eskin DG, Suyitno, Katgerman L. Mechanical properties in the semisolid state and hot tearing of aluminium alloys. Prog Mater Sci 2004;49(5):629-711.

[5] Gourlay CM, Dahle AK. Dilatant shear bands in solidifying metals. Nature 2007;445(7123):70-3.

[6] Gourlay CM, Dahle AK, Nagira T, Nakatsuka N, Nogita K, Uesugi $\mathrm{K}$, et al. Granular deformation mechanisms in semi-solid alloys. Acta Mater 2011;59(12):4933-43.

[7] Mathier V, Jacot A, Rappaz M. Coalescence of equiaxed grains during solidification. Modell Simul Mater Sci Eng 2004;12(3): 479-90.

[8] Vernède S, Rappaz M. A simple and efficient model for mesoscale solidification simulation of globular grain structures. Acta Mater 2007;55(5):1703-10.

[9] Phillion AB, Cockcroft SL, Lee PD. A three-phase simulation of the effect of microstructural features on semi-solid tensile deformation. Acta Mater 2008;56(16):4328-38.

[10] Sun Z, Logé R, Bernacki M. 3d finite element model of semi-solid permeability in an equiaxed granular structure. Comput Mater Sci 2010;49(1):158-70.

[11] Sistaninia M, Phillion AB, Drezet JM, Rappaz M. 3d granular model of semi-solid metallic alloys undergoing solidification: fluid-flow and localization of feeding. Acta Mater 2012;60:3902-11.

[12] Sistaninia M, Phillion A, Drezet JM, Rappaz M. Simulation of semisolid material mechanical behavior using a combined discrete/finite element method. Metall Mater Trans A 2011;42:239-48.

[13] Yuan L, O'Sullivan C, Gourlay C. Exploring dendrite coherency with the discrete element method. Acta Mater 2012;60(3):1334-45.

[14] Monroe C, Beckermann C. Development of a hot tear indicator for steel castings. Mater Sci Eng A 2005;413-414:30-6.

[15] M'Hamdi M, Mo A, Fjaer HG. Tearsim: a two-phase model addressing hot tearing formation during aluminum direct chill casting. Metall Mater Trans A 2006;37A(10):3069-83.

[16] Mathier V, Vernède S, Jarry P, Rappaz M. Two-phase modeling of hot tearing in aluminum alloys: applications of a semicoupled method. Metall Mater Trans A 2009;40:943-57.

[17] Bernard D, Nielsen O, Salvo L, Cloetens P. Permeability assessment by $3 \mathrm{~d}$ interdendritic flow simulations on microtomography mappings of Al-Cu alloys. Mater Sci Eng A 2005;392(1-2):112-20.

[18] Terzi S, Salvo L, Suéry M, Limodin N, Adrien J, Maire E, et al. In situ x-ray tomography observation of inhomogeneous deformation in semi-solid aluminium alloys. Scripta Mater 2009;61(5): 449-52.

[19] Phillion A, Cockcroft S, Lee P. X-ray micro-tomographic observations of hot tear damage in an $\mathrm{Al}-\mathrm{Mg}$ commercial alloy. Scripta Mater 2006;55(5):489-92. 
[20] Fuloria D, Lee P. An X-ray microtomographic and finite element modeling approach for the prediction of semi-solid deformation behaviour in Al-Cu alloys. Acta Mater 2009;57(18):5554-62.

[21] Ludwig O, Drezet JM, Martin C, Suéry M. Rheological behavior of $\mathrm{Al}-\mathrm{Cu}$ alloys during solidification constitutive modeling, experimental identification, and numerical study. Metall Mater Trans A 2005;36(6):1525-35.

[22] Braccini M, Martin C, Tourabi A, Bréchet Y, Suéry M. Low shear rate behavior at high solid fractions of partially solidified Al-8 wt.\% $\mathrm{Cu}$ alloys. Mater Sci Eng A 2002;337(12):1-11.

[23] Mathier V, Grasso PD, Rappaz M. A new tensile test for aluminum alloys in the mushy state: experimental method and numerical modeling. Metall Mater Trans A Phys Metall Mater Sci 2008;39 A(6):1399-409.

[24] Flemings M, Metz S. Fundamental study of hot tearing. In: Trans Amer Foundrymen's soc, vol. 78; 1970. p. 453-60.

[25] Phillion A, Hamilton R, Fuloria D, Leung A, Rockett P, Connolley $\mathrm{T}$, et al. In situ X-ray observation of semi-solid deformation and failure in Al-Cu alloys. Acta Mater 2011;59(4):1436-44.

[26] Dantzig JA, Rappaz M. Solidification. EPFL Press; 2009.

[27] Sistaninia M, Phillion A, Drezet JM, Rappaz M. Simulation of semi-solid material behaviour using a discrete finite element method. In: 49th annual conference of metallurgists, Vancouver, BC; 2010 . 